\title{
Study on Mathematical Model of Compression Performance of Bamboo Culm Integrated Bamboo Bundles Glulam Beam
}

\author{
Zhangrong Zhao ${ }^{1}$, Xiaomei Sui ${ }^{2, *}$, Xingyu Liu $^{3}$, Weihua Sun ${ }^{1}$ and Xiangling Gong ${ }^{1}$ \\ ${ }^{1}$ Department of Logistics, Beijing WUZI University, Beijing, 101149, China \\ ${ }^{2}$ Department of electronic and Information Engineering, North China Institute of Science \&Technology, Sanhe, 065201, China \\ ${ }^{3}$ Guolian Automotive Power Battery Research Institute Co., Ltd., Beijing, 100083, China \\ *Corresponding author
}

\begin{abstract}
This paper proposes a new type of the bamboo culm integrated bamboo bundles glulam using bamboo culm as skeleton, which solves the problem of the irregular size of the original bamboo and the hollow structures. A three dimension finite element model of bamboo culm integrated bamboo bundles glulam was established using continuum damage mechanics and cohesive element model. This failure and damage evolution process of bamboo culm integrated bamboo bundles glulam under the compression load was analyzed, and the process of adhesive failure between bamboo bundles was also analyzed. The results showed that $90^{\circ}$ bamboo bundles layer were damaged, and $0^{0}$ bamboo bundles layer were not damage. The load capacity of bamboo laminated glue material with the skeleton is 6 times higher than bamboo laminated glue material.
\end{abstract}

Keywords-bamboo culm integrated bamboo bundle; glulam; failure; finite element

\section{INTRODUCTION}

With modern industry development, people use a large amount of non-renewable materials as production materials, causing bad effects on human ecology. In modern buildings industry high-energy-consuming raw materials such as steel bars, concrete, and cement currently are used. At the same time, these raw materials cannot be regenerated, it is an unsustainable production patterns. Bamboo is a kind of short growth cycle green material. It has good tensile and compressive properties and is very suitable for the building beams because it's longitudinal fiber structure. It is difficult to apply the original bamboo to industrial building material for its natural irregular size. In order to realization of bamboo industrial application, it is necessary to reorganize and modify the natural bamboo. At present, there are many methods about reorganized bamboo and laminated bamboo.

The industrial utilization of bamboo in the prior art is the reorganization of the bamboo bundles splitted by bamboo piece, or the reorganization using the natural bamboo culm. However, there is no method to combine the bamboo bundle with the bamboo culm to form the reorganized material. The authors studied a bamboo reorganized material, which included the bamboo bundles obtained by rolling, directionally flattening and parsing the original bamboo culm, and a bamboo culm as skeleton with the natural hollow structure. The bamboo bundles on which the adhesive was disposed were put at the bottom and the top layer, the bamboo culm as skeleton coated with an adhesive was put in the middle of material, and the gaps between the bamboo skeletons were filled with the bamboo bundles.

The bamboo glulam not only makes full use of the high strength and high elasticity of the natural physical bamboo culm structure, but also absorbs the forming advantages of the modified bamboo bundles. It realizes the shape rule and strength dual target. A bamboo culm is set in the bamboo bundles; those have two different shapes but the same material. Compared other composites with different material, the bonding strength of this material is higher, and the performance is stable under the change of the temperature. The bamboo reorganization material can be applied to a variety of architectural beams and columns, wall surfaces, door panels, and furniture floors and panels. It has a good market development prospect, expands the application of bamboo materials, and increases the economic value of bamboo products. The design model is shown in Figure 1.

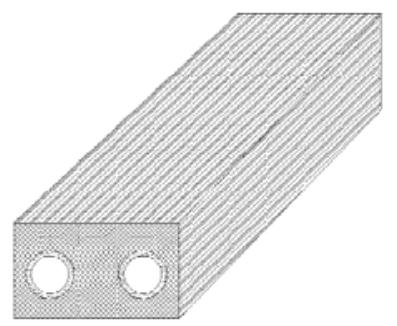

FIGURE I. BAMBOO CULM INTEGRATED BAMBOO BUNDLES GLULAM

The performance of this glulam depends on the properties of the bamboo culm as a skeleton and the gluing properties of the bamboo bundles, and the gluing properties of the bamboo culm and bamboo bundles play a decisive role in this glulam. It is an excellent choice to study bamboo glulam by numerical simulation. In order to solve those problems, the research group established a numerical model of composite bamboo bundle glulam, and studied the cracking of bamboo bundles, skeletons and failure under the compression load. In the bamboo glulam manufacturing process parameters on the material performance, 
many scholars have done research. Matthew Penellum ${ }^{[1]}$ study fiber volume fraction of laminated bamboo on bending stiffness. Zujian Huang [2] study bamboo material parameters on the bamboo-based panel. For bamboo reorganization and glulam, many scholars have carried on the research. Compared with structural wood, reorganized bamboo material has the ability to carry large bending compressive loads and good mechanical properties ${ }^{[3-5]}$. Building structure beam bends under the load, the reinforced concrete beam only depends on the reinforcement to bear the tensile stress, but the bamboo longitudinal fiber structure causes the fibers under the axis to bear the tensile load, so the bearing capacity is strong. In the design of steel structures and concrete structures fields, finite element mathematical models have been successfully used to optimize structural design parameters. Some scholars have used finite element analysis to analyze the design of wood structures [6, 7]. However, there are few reports on the application of finite element methods in bamboo reorganized material applications. In this paper, a three-dimensional finite element model was established for the newly bamboo culm composite bamboo bundles beam proposed, and the material failure process under pressure was analyzed. It provides a certain support for the application of the material.

\section{Establishment OF A MATHEMATICAL MODEL}

The bamboo culm integrated bamboo bundles glulam can be regarded as a multilayer laminated composite material and is an anisotropic orthogonal composite material with bidirectional fiber distribution. It is also different from wood and its composite materials. The maximum stress theory, Tsai-Wu theory, and Tsai-Hill theory are often used for the destruction of wood and composite materials. These theories are mostly used for the analysis of unidirectional lamination materials and rarely used in bidirectional fiber composites. Because the bamboo bundles of the bamboo culm integrated bamboo bundles glulam are similar to composite materials, the Hashin criterion is used to determine whether the material is damaged or not. The in-plane damage model was established based on the Hashin criterion and the damage mechanics of continuous media. The theory of progressive damage analysis was used to simulate the initiation and evolution of in-plane damage. Seven different modes of damage failure including fiber tension (or compression) fracture, matrix tension and shear fracture in transverse, matrix compression and shear in transverse, matrix layering under parallel tension and shear. Compression failure of fiber of bamboo bundles,

$$
\left(\frac{\sigma_{11}}{X_{C}}\right)^{2} \geq 1
$$

In the equation, $\sigma_{11}, X_{C}$ are the tensile stress in the $\mathrm{X}$ direction of the fiber axis and the compressive strength in the $\mathrm{X}$ direction of the fiber axis. Compression failure in the matrix of bamboo bundles,

$$
\left(\frac{\sigma_{22}}{Y_{C}}\right)^{2}+\left(\frac{\tau_{12}}{S_{12}}\right)^{2}+\left(\frac{\tau_{23}}{S_{23}}\right)^{2} \geq 1
$$

In the equation, $\sigma_{22}, \tau_{12}, \tau_{23}, Y_{c}, S_{23}$ are $\mathrm{Y}$ direction tensile stress of the matrix, $X-Y$ plane shear stress of the matrix, Y-Z plane shear stress of the matrix-direction compressive strength and Y-Z plane shear strength, respectively.

The glue layer between bamboo bundles will also be damaged under load. The adhesive layer was modeled with a certain thickness of cohesive elements, and a zero-thickness cohesive element was used for the layer between the adhesive layer and the bamboo bundle layer. A bilinear constitutive relational model was used for cohesive element degradation. For the damaged layer, linear elasticity relationship was used when no damage has occurred at the beginning.

\section{Finite ElEMENT MODEl CALCULATION RESUlTS AND ANALYSIS}

Using the in-plane and between layer damage models previously established, a three dimension finite element model of the bamboo culm integrated bamboo bundles glulam was realized in ABAQUS, and the model was used to analyze the damage of this material under pressure loading. The boundary condition of the model was that the compressive displacement load was applied to both ends until the material was failure. Because of the symmetry of the model, a quarter-model calculation was chosen to reduce calculations. The bamboo culm as a skeleton was simulated using a linear elastic model. The cohesive layer between the bamboo culm and the bamboo bundles was modeled with a cohesive element. The bamboo bundle layer was modeled with a composite material model, and the adhesion layer between the bamboo bundle plies was simulated with a cohesive element. Displacement and boundary conditions are shown in Figure 1.a. The overall model established is shown in Figure 1.b. $0^{0}$ first layer, $90^{\circ}$ first layer, $0^{0}$ second layer, $90^{\circ}$ second layer of bamboo bundles are laid along the positive direction of the $\mathrm{Y}$ axis, respectively. The bamboo culm is placed at the center. The sizes of the model were: $72 \mathrm{~mm} \times 80 \mathrm{~mm} \times 200 \mathrm{~mm}$. The bamboo culm is $16 \mathrm{~mm}$ in outer diameter, $6 \mathrm{~mm}$ in inner diameter, and $100 \mathrm{~mm}$ in length. Bamboo bundles thickness is $5 \mathrm{~mm}$, glue thickness is $2 \mathrm{~mm}$. The bamboo bundles layer adopts 8-node continuous shell element SC8R, and the adhesive layer thickness is $0.1 \mathrm{~mm}$ cohesive element COH3D8. The four layers are arranged according to $0^{0} / 90^{\circ} / 0^{0} / 90^{\circ}$. The model established is shown in Figure 2. 

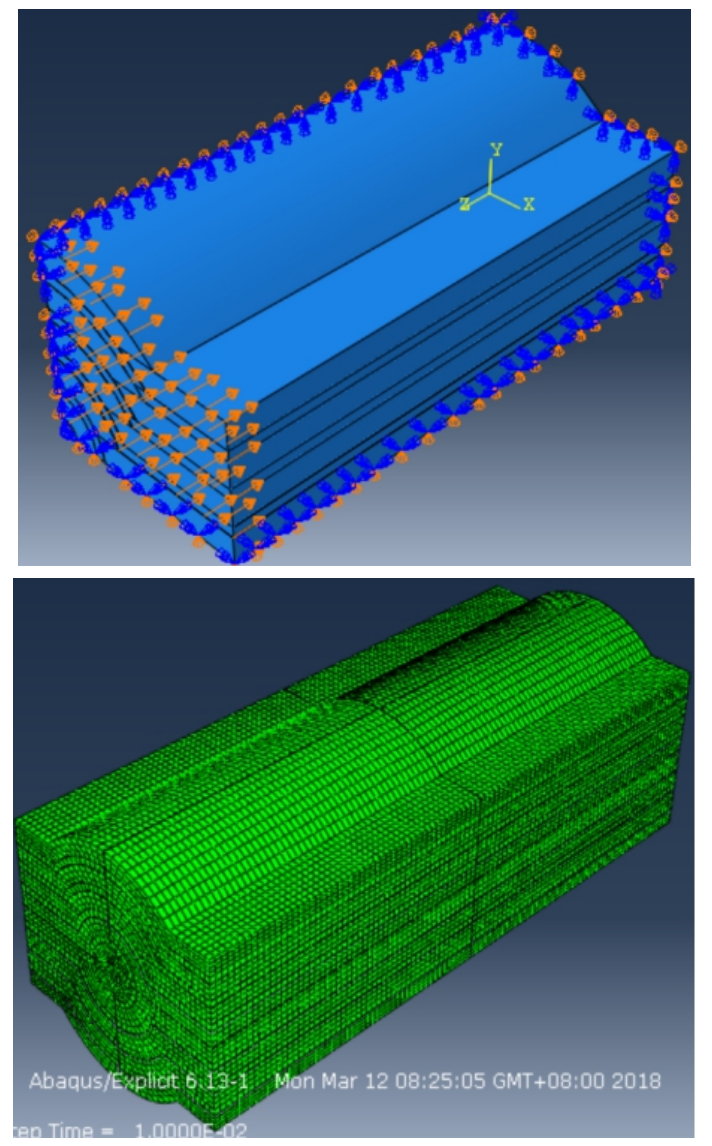

FIGURE II. FINITE ELEMENT MODEL ESTABLISHED

\section{A. Failure Mode in Lamination}

In order to study the damage of the bamboo culm integrated bamboo bundles glulam under load, 10 elements were selected along the Z-axis to study the variation of the stress changes with time. The stress changes with time is shown in figure 3,4 . From figure1, it can be seen that the stress of 10 elements in the first and second layer $0^{0}$ ply changes with time. There have the same variation trend at different positions, and the stress first increases with time, then reaches the peak value of $14 \mathrm{MPa}$ at 0.009s, drops to $10 \mathrm{MPa}$.

This is due to a certain amount of plastic deformation of the material at that moment. For the second layer $0^{0}$ ply of bamboo bundles, 10 elements at the same position were selected to see the stress changes with time. It can be seen that the stress reaches $11 \mathrm{MPa}$ at $0.008 \mathrm{~s}$ and drops to $2 \mathrm{MPa}$ at last. The plastic deformation occurs in bamboo bundles. 10 elements of the first layer $90^{\circ}$ ply were selected at the same position to see the stress change with time. It can be seen that the material stress reaches a peak value of $42 \mathrm{MPa}$ at $0.008 \mathrm{~s}$ and drops to 0 with time, and the material plastic deformation and failure occurs in this ply. For the second layer $90^{\circ}$ ply of bamboo bundles, 10 elements were selected at the same position to see the change in stress with time. It can be seen that the material stress reaches the maximum value of $42 \mathrm{MPa}$ at $0.008 \mathrm{~s}$ and drops to 0 at last, the plastic deformation and failure occurs in the ply. The stress change curve is the same as the first layer
$90^{0}$ ply of bamboo bundles. From the stress changes in the figures, it can be seen that the bamboo culm integrated bamboo bundles glulam $90^{\circ}$ layups will fail and $0^{0}$ layup only produces a small amount of plastic strain under the compression displacement load.

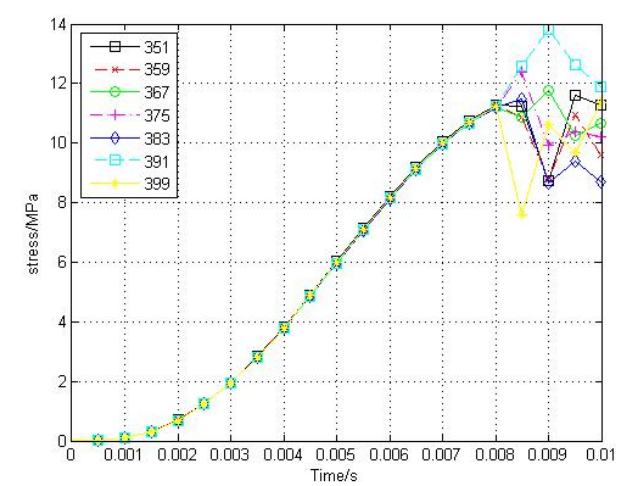

(A)

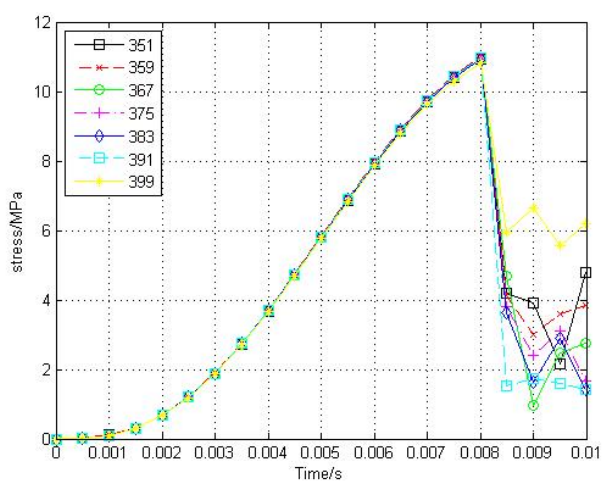

(B)

FIGURE III. STRESS IN THE BUNDLES OF THE MATERIAL CHANGES WITH TIME (A) $0^{\circ}$ FIRST LAYER, (B) $0^{\circ}$ SECOND LAYER 


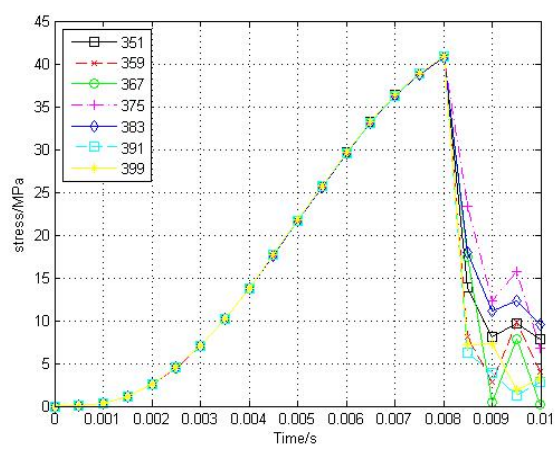

(A)

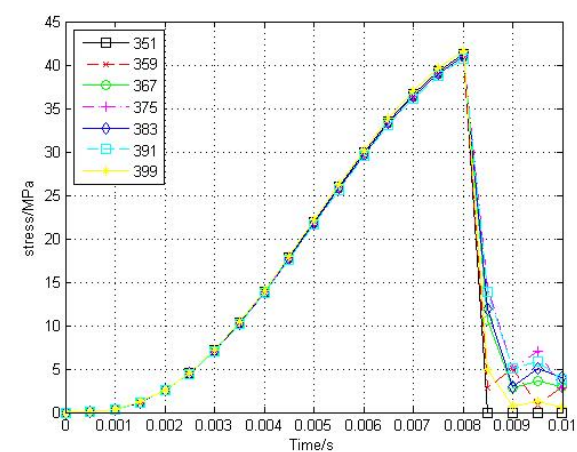

(B)

FIGURE IV. STRESS IN THE BUNDLES OF LAMINATED BAMBOO BUNDLES CHANGES WITH TIME (A) $90^{\circ}$ FIRST LAYER, (B) $90^{\circ}$ SECOND LAYER

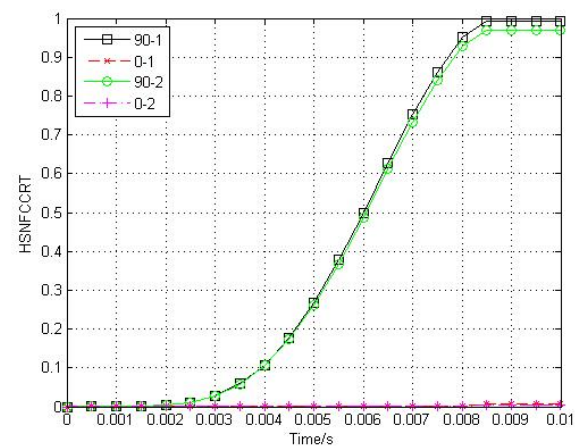

(A)

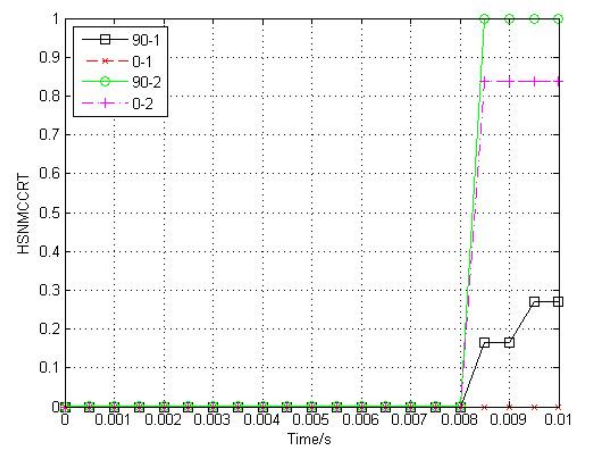

(B)

FIGURE V. FIBER FAILURE CHANGE WITH TIME (A) FIBER TENSION FAILURE, (B) FIBER COMPRESSION FAILURE
From figure 5, it can be seen that the main failure of the bamboo culm integrated bamboo bundles glulam is the damage of the bamboo bundles, which is shown in the tensile failure of the bamboo bundle fiber and the failure of the matrix. By analyzing the failure process of the material, it can be seen that the failure of the fiber compression damage in the material mainly laid in the $90^{\circ}$ plies 1 and 2 of bamboo bundles, the damage occurred in $0.0002 \mathrm{~s}$, and reached $100 \%$ at the time of $0.0085 \mathrm{~s}$. There was no damage to the $0^{\circ}$ bamboo plies 1 and 2 plies. From the failure results of the compression failure of the material matrix, it can be seen that the matrix in the $0^{0}$ ply 1 and the $90^{\circ}$ ply 2 were damaged. The matrix in $90^{\circ}$ ply 2 began to be damaged at the time of $0.008 \mathrm{~s}$, and the damage reached $100 \%$ at $0.0082 \mathrm{~s}$, and the matrix in $0^{0}$ ply 1 damage reached $85 \%$ at 0.0085 s. There was no damage in $0^{\circ}$ ply 2 and $90^{\circ}$ ply 1 .

\section{B. Failure Between Layers}

In addition to the failure of compression of bamboo fiber bundles in bamboo bundles and the failure of compression of the matrix, the failure of the bamboo culm integrated bamboo bundles glulam can also be failure between bamboo bundles. Once failure occurs between the bamboo bundle layers, the material fails. A mathematical model that describes the layering of bamboo bundles is established in this section. The size of the model was a $72 \mathrm{~mm} \times 80 \mathrm{~mm} \times 200 \mathrm{~mm}$ bamboo bundle layer. The outer dimension of the glue layer was the same as that of the layup, and the thickness was $0.2 \mathrm{~mm}$. The adhesive layer between bundles layer was simulated with a certain thickness of cohesive element, and the bilinear equation was used for the cohesive element degradation. A linear elasticity was used for the adhesive layer between bundles layer when no damage has occurred at the beginning. When the inner tensile stress and the shear stress of the adhesive layer reached the tensile stress strength and shear strength, the adhesive layer cracked. In this paper, the secondary stress criterion is applied by considering the internal tensile stress and shear stress mixing effect.

In ABAQUS, a three dimension finite element model of the bamboo culm integrated bamboo bundles glulam considering the layering of bamboo bundle layers was established. In order to accurately simulate layering, the adhesive layer was subdivided into a layer of mesh along the thickness direction, and a reduced-integration 8-node continuous shell element SC8R was used. Cohesion element COH3D8 with a thickness of $0.2 \mathrm{~mm}$ was used. The sample is axisymmetric, only the $1 / 4$ model is analyzed, and one end of the model applies a compressive displacement load. 


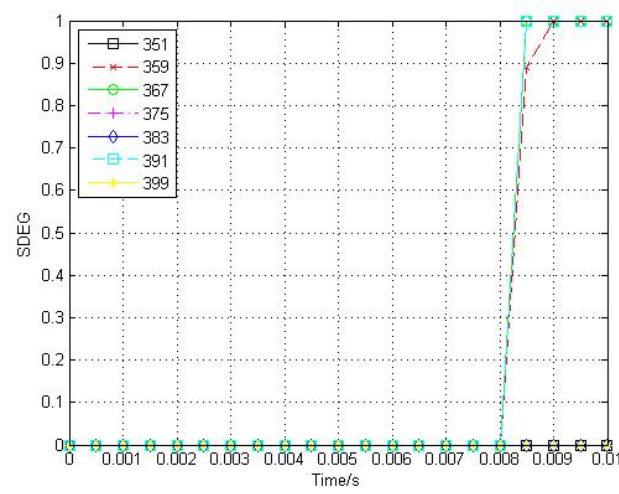

(A)

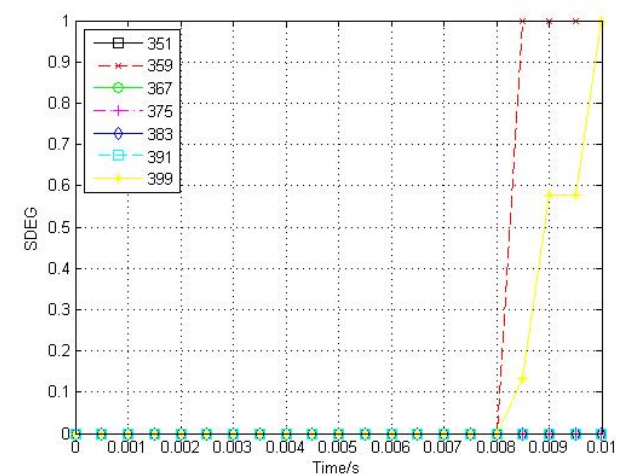

(B)

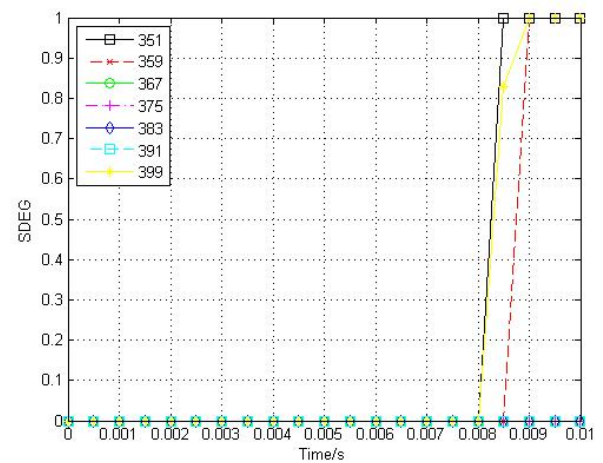

(C)

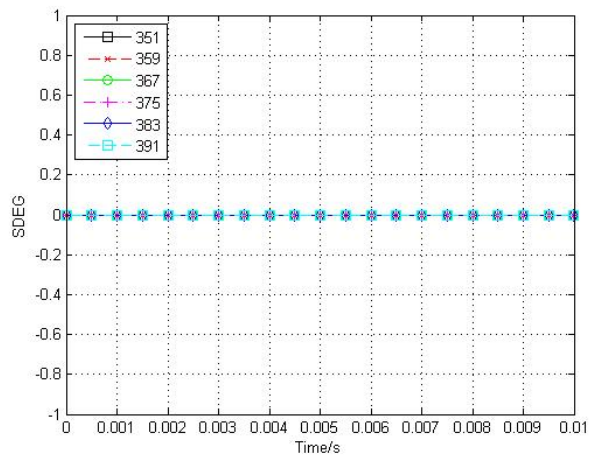

(D)

FIGURE VI. THE STIFFNESS DEGRADATION COEFFICIENT OF EACH LAYER CHANGES WITH TIME (A) FIRST LAYER, (B) SECOND LAYER, (C) FIRST LAYER, (D) SECOND LAYER
The changes in stiffness degradation coefficient with time for different layers are shown in Figure 6. It can see that the first, second, and third layers of the adhesive layer cracked, and the fourth layer did not crack. 7 elements of the adhesive layer are taken to observe cracking of the adhesive layer. The cracks appeared in the adhesive layer, and only two of the seven elements selected in the first adhesive layer began cracking at $0.008 \mathrm{~s}$. The stiffness degradation coefficient reached $100 \%$ at $0.0085 \mathrm{~s}$. Only one of the seven elements selected for the second adhesive layer began to crack at $0.008 \mathrm{~s}$, and the stiffness degradation factor reaches $100 \%$ at 0.0085 s. Three of the seven elements selected in the third adhesive layer were degraded. The time of occurrence was at $0.0085 \mathrm{~S}$. The complete cracking coefficient of one element reached $100 \%$ at the time of $0.0085 \mathrm{~s}$, and the two elements completely cracked at $0.009 \mathrm{~s}$. There was no damage for the adhesive layer glued to the bamboo culm, the coefficient of stiffness degradation was 0 , and no cracking occurred.

\section{Performance Comparison with Bamboo Bundles}

Comparing the compressive performance of the bamboo culm integrated bamboo bundles glulam and the solid bamboo bundle glulam. The bamboo culm integrated bamboo bundles glulam has the bamboo culm as central skeleton, the solid bamboo bundle glulam only has the same thickness bamboo bundles layer and the glue layer. Those two materials are all applied displacement load until failure.
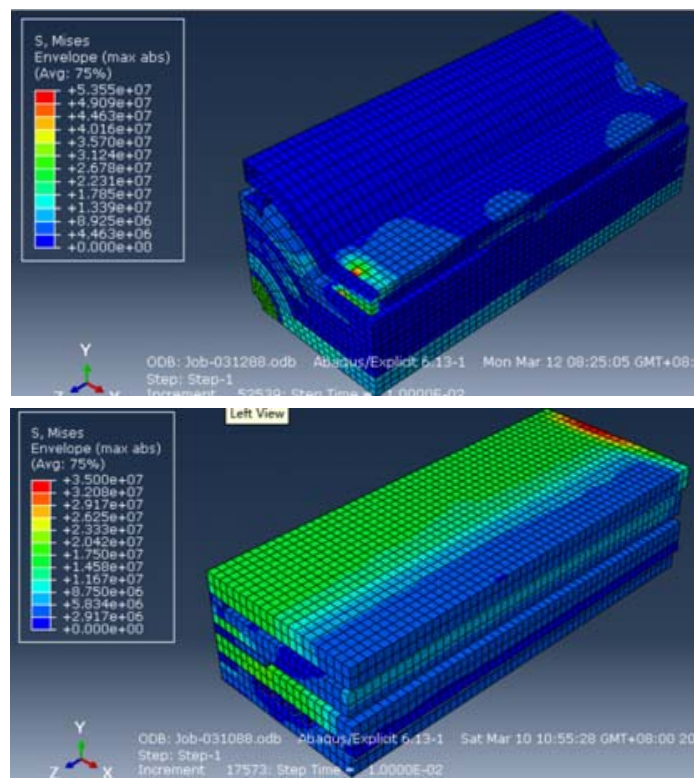

FIGURE VII. DISTRIBUTION OF COMPRESSIVE STRESS OF THE BAMBOO CULM INTEGRATED BAMBOO BUNDLES GLULAM AND BAMBOO BUNDLES GLULAM (A) THE BAMBOO CULM INTEGRATED BAMBOO BUNDLES GLULAM, (B) BAMBOO BUNDLES GLULAM

As shown in Figure 7.A, the bamboo culm integrated bamboo bundles glulam fails under the pressure displacement of $0.000249 \mathrm{~m}$, the maximum stress reaches 53MPa, and the outermost bamboo bundles layer fails, and the first to third layers of adhesive layer from the outside to the inside are all cracking. The bamboo bundles glulam stress are shown in Fig. 
7.B, failure at a pressure displacement of $0.00154 \mathrm{~m}$ occurs, the maximum stress reaches $35 \mathrm{MPa}$, and the outermost layer of bamboo bundles fails, cracking occurs from the first to third layers of glue from the outside to the inside. The compressive strength of the bamboo culm integrated bamboo bundles glulam is six times than the solid bamboo bundles glulam. This is because the bamboo culm skeleton plays a major supporting role. At the same time, it was found that for the bamboo culm integrated bamboo bundles glulam the bamboo bundles on the outermost and adjacent bamboo culm are subject to greater stress. For solid bamboo bundles glulam two $90^{\circ}$ layer withstands more stress.

\section{CONCLUSION}

In this paper, a three dimension finite element model of the bamboo culm integrated bamboo bundles glulam under pressure is established. By comparison between the bamboo culm integrated bamboo bundles glulam and bamboo bundles glulam, the following conclusions are obtained.

1) Under compressive load, failure of all the fibers in the ply 1and ply 2 of the $90^{\circ}$ bamboo bundles occur, and failure of the fibers in ply 1 and ply 2 of the $0^{0}$ bamboo bundles do not occur.

2) Under the compressive load, failure of the matrix in $90^{\circ}$ bamboo bundle ply 2 and $0^{0}$ bamboo bundle ply 2 occur, failure of the matrix in $90^{\circ}$ bamboo ply 1 and $0^{\circ}$ bamboo bundle ply 1 do not occur.

3) In the four adhesive layers, three adhesive layers except the adhesive layer for the bamboo culm are all exits cracking. Compared the bamboo culm integrated bamboo bundles glulam with the bamboo bundles glulam, the compressive performance of the bamboo culm integrated bamboo bundles glulam is increased by 6 times. The failure of the bamboo culm integrated bamboo bundles glulam mainly occurs in the outermost $90^{\circ}$ bamboo bundle ply 2, and for the bamboo bundles glulam the main failure occurs in $90^{\circ}$ bamboo bundle ply 2 and ply 1.

\section{ACKNOWLEDGMENT}

This research was financially supported by National Natural Science Foundation of China (No.31470588) Science and technology project of Hebei Province(No.15211830.

\section{REFERENCES}

[1] Matthew Penellum, Bhavna Sharma, Darshil U. Shah, etc., "Relationship of structure and stiffness in laminated bamboo composites," Construction and Building Materials, vol. 165, pp. 241-246, April 2018.

[2] Zujian Huang, Yimin Sun, Florian Musso. "Experimental study on bamboo hygrothermal properties and the impact of bamboo-based panel process" Construction and Building Materials, vol.155, pp. 1112-1125, August 2017.

[3] Juan F. Correal, Juan S. Echeverry, etc., "Experimental evaluation of physical and mechanical properties of glued laminated Guadua angustifolia Kunth,”Construction and Building Materials, vol. 73, pp. 105-112, June 2014.

[4] Su Yi, Zong Sheng Jing, Xu Dan Huang, etc., "Experimental study on flexural behavior of bamboo composite,” Journal of Building Science and Engineering, vol. 01, pp. 54-60, April 2016.
[5] Li Haitao, Zhang Qisheng, Wu Gang, etc., "Research progress of bamboo Glulam,” Journal of Forestry Engineering, vol.06, pp. 10-16, March 2016.

[6] Zhou Junwen, Shen Yurong, "Numerical Analysis on flexural bearing capacity of reorganized bamboo beams," Science and Technology of China, vol. 3, pp. 10-16,June 2018.

[7] Shandy Alexandra Morassi, Júlia Lopes Ribeiro da Silva, “Altibano Ortenzi, omparative result between standard requirements and finite element analysis of wood-frame panels,” Journal of Building Engineering, vol. 15, pp. 78-84, August 2018.

[8] B. Toson, P. Viot, J. J. Pesqué, "Finite element modeling of Balsa wood structures under severe loadings,” Engineering Structures, vol. 70, pp. 36-52, June 2014. 